\title{
Germanica
}

GERMANICA

5 | 1989

Le mal d'être

\section{Ces routes qui sont des déroutes}

À propos du roman Brandung de Martin Walser

Wege sind Abwege. Zu Martin Walsers Roman Brandung

\section{Erika Tunner}

\section{(2) OpenEdition}

\section{Journals}

Édition électronique

URL : http://journals.openedition.org/germanica/2634

DOI : 10.4000/germanica.2634

ISSN : 2107-0784

Éditeur

Université de Lille

Édition imprimée

Date de publication : 31 décembre 1989

Pagination : 159-166

ISSN : 0984-2632

Référence électronique

Erika Tunner, "Ces routes qui sont des déroutes », Germanica [En ligne], 5 | 1989, mis en ligne le 09 février 2015, consulté le 06 octobre 2020. URL : http://journals.openedition.org/germanica/2634 DOI : https://doi.org/10.4000/germanica.2634

Ce document a été généré automatiquement le 6 octobre 2020.

(c) Tous droits réservés 


\title{
Ces routes qui sont des déroutes
}

\author{
À propos du roman Brandung de Martin Walser \\ Wege sind Abwege. Zu Martin Walsers Roman Brandung
}

Erika Tunner

1 Martin Walser est bien connu pour le regard critique qu'il porte sur la société allemande d'après-guerre et pour la verve satirique avec laquelle il sait dénoncer les travers de notre temps. Dans le roman Brandung ${ }^{1}$ (Ressac) (1985), c'est sur la côte californienne que se déroule une histoire apparemment banale. Un professeur allemand s'éprend d'une jeune étudiante américaine, vit une passion sans espoir, puis regagne son pays pour y retrouver une existence morose. Le véritable sujet du livre cependant est le mal d'être: il se manifeste à des degrés divers, dans un milieu universitaire, milieu de compétition et de rivalités en même temps que dispensateur de privilèges, où les activités et les loisirs sont façonnés par le monde d'aujourd'hui, par sa science, sa technique, ses modes et ses dadas.

2 Helmut Halm est marié et père de deux filles, Lena et Juliane, dont l'une et l'autre ont des problèmes sentimentaux. Son ménage ne lui procure guère de folles joies, son métier non plus. Il enseigne dans un lycée. Son supérieur, le directeur adjoint, un homme du genre "J'arrive-à-tout-avec-tout-le-monde", grimpe allègrement les échelons sociaux, assisté d'une épouse qui écrit entre trois et cinq livres pas an, tandis que Sabine, la femme de Halm, ne fait rien de spectaculaire. Dans la vie de Halm, il n'y a pas de choix véritable, pas de rupture non plus. Le calme plat ou presque. Aucune raison majeure de ressentir un mal d'être, et du reste Halm ne le ressent pas.

3 Vraiment pas? Un jour, un camarade d'étude, Rainer Mersjohann, appelle de Oakland, Californie. Rainer Mersjohann, professeur à l'Université, chairman depuis quelques années, invite son ami pour le semestre d'automne. Halm aurait deux cours d'allemand à assurer. Une occasion exceptionnelle, un emploi pour lequel les candidats se bousculeraient aux États-Unis, paraît-il.

4 Halm part avec sa femme et sa fille Lena. Secrètement, il attend un renouvellement, une expérience forte qui le changerait, une sensation violente qui l'emporterait. La 
platitude quotidienne sera balayée par les grandes vagues, le ressac, l'Océan, le Pacifique.

5 Au premier plan il y a donc Halm. 55 ans. Un âge où on vous dit : plus très jeune, non, mais pas encore vieux. Un homme mûr, selon la formule. «Keep your age a secret» est un des slogans que Halm entendra souvent aux États-Unis. Au campus d'Oakland arrive ce qui devait arriver et qui ne surprendra personne: Halm tombe amoureux d'une étudiante (de l'âge de sa fille Lena) que les collègues surnomment la «ravissante idiote ». Ravissante sûrement, elle plaît à un jeune sportif, vedette de l'équipe de waterpolo, un "all-american boy ", qu'elle préfère à tous les professeurs d'Université, en quoi elle manque incontestablement de respect et de considération. Une idiote? Halm discute avec elle des poèmes de Rilke, des sonnets et des drames de Shakespeare, c'est tout juste s'il ne la trouve pas éblouissante d'intelligence. Rien de bien original dans tout cela. Aussi n'est-ce pas en attendant la grande aventure (qui, du reste, ne se produira pas) que le lecteur continue de tourner les pages. C'est qu'il y a Rainer Mersjohann.

Rainer Mersjohann, le pur, le juste, l'intransigeant. Rainer Mersjohann, le professeur désabusé, le mari humilié, l'homme aux abois. À l'âge de vingt ans, il avait publié ses sonnets dont ses professeurs donnaient lecture dans leurs séminaires. Un esprit brillant, disaient-ils, un jeune génie, quel avenir plein de promesses, quel espoir pour les lettres! Puis le jeune génie prit le large, il disparut, on ne savait pas où. Bien plus tard, Halm reçut de lui quelques lettres, des cartes de vœux, pas de correspondance régulière, mais des signes de vie. Rainer Mersjohann s'était installé dans la «Terre Promise ». C'est là que Halm le revoit, vingt-cinq ans plus tard.

7 Les Halm descendent de l'avion. Ils cherchent du regard Rainer Mersjohann, grand, mince, une mèche blonde, des yeux d'un éclat profond, en un mot : Ariel. Il a du retard, un empêchement, il n'a jamais su être à l'heure. Au bout d'un quart de siècle et de milliers de kilomètres Halm attend Mersjohann et Mersjohann n'est pas au rendezvous... C'est que Halm cherche Ariel et que c'est Falstaff qui se présente. Falstaff voulait qu'on le prît pour Mersjohann. Bien sûr, Ariel et Falstaff, cela fait deux. Rainer Mersjohann l'ancien et le nouveau Rainer Mersjohann, cela ne faisait pas deux, c'était bel et bien la même personne. Plus d'allure de poète, plus de cils dorés; un homme gros, les joues pendantes, un double menton, une barbe broussailleuse, un crâne chauve, un regard incertain. Il fallait s'y faire.

Rainer Mersjohann ou le mal d'être.

Lorsque Mersjohann installe les Halm dans la maison d'un collège parti en mission, Helmut Halm découvre un Mersjohann bricoleur, développant un sens étonnemment pratique, mettant en route, ici et là, de petites choses tombées en panne, travaillant sans hâte, d'un air reposé, ignorant le stress et toutes ses conséquences. Mais il voit aussi un Mersjohann aimant la bouteille et habitué à la boisson. L'alcoolisme n'est pas toujours affaire d'hérédité, d'épicurisme ou d'indiscipline personnelle. C'est aussi, comme toutes les drogues, un palliatif à un mal. C'est aussi un moyen d'autodestruction et Mersjohann sait que l'intoxication progressive mettra fin un jour à cette lucidité aiguë avec laquelle il observe sa vie allant de crise en crise et qu'il n'arrive plus ni à maîtriser ni même à structurer. Ceux qui n'ont jamais bu n'éprouvent qu'une vague pitié pour les individus dont ils jugent les goûts étrangement singuliers : un chat qui adore la viande ne comprendra pas pourquoi on lui offre une orange pelée qu'il déteste. Ceux qui boivent modérément ont tendance à manifester du mépris face à la démesure, 
symptôme d'un manque déplorable de force et de contenance. Seule Elissa, la femme de Mersjohann, a parfaitement conscience des intentions suicidaires de son mari : « il est en train de se tuer » dit-elle à Halm, avec un de ces sourires « décoratifs » dont elle a le secret.

Pourtant, Rainer Mersjohann assure ses cours, fait des conférences, fréquente ses amis, donne des soirées... Mais il est arrivé à un point de non-retour où le mal d'être qui le ronge aura raison de lui. Martin Walser nous en montre les causes, habilement, comme si de rien n'était, en bavardant pendant des pages et des pages, en passant d'une réception mondaine à l'autre, sur un ton allègre d'une ironie feutrée.

La vie privée, le travail, le pays. Dans le cas de Mersjohann ils ne forment pas seulement un tout qu'on ne peut pas séparer, ils constituent le piège dans lequel il est pris.

Elissa d'abord. Une descendante des premiers chercheurs d'or. Une femme belle, sûre d'elle-même, aimant l'insolite. De ses deux fils, le lecteur ne connaît que les photos : Milton et Jamey, « ils ressemblaient tous les deux à de jeunes Rainer » - mais Rainer ne s'y reconnaît pas : "Milton et Jamey me ressemblent aussi peu que je me ressemble moi-même », dit-il. Elissa s'absente souvent et elle ne s'absente pas toujours seule. Sa dernière conquête, un homme de couleur, directeur du département d'art dramatique, lui fait ouvertement la cour, sous les yeux de son mari, au beau milieu d'une société rayonnante de bonheur. Halm, nous dit l'auteur, ne se rappelait pas avoir jamais eu autant de plaisir à contempler une assemblée de gens aussi bien habillés, à fréquenter des lieux où régnait tant de vivacité, de légèreté et d'aisance, sur le fond d'un décor féerique - une maison sur les hauteurs, avec vue sur San Francisco, sur la baie lumineuse, sur le Golden Gate Bridge. Halm s'exclame qu'il est impossible de concevoir une journée plus belle. Mais des doutes commencent à surgir : n'était-ce pas aussi une journée on ne peut plus pénible?

13 Mersjohann s'était mis à déraper. Il ne supporte pas la trahison, en quoi, naturellement, il a grand tort, la trahison étant la source de beaucoup de victoires et de succès insoupçonnés. La trahison d'Elissa, douloureuse en l'occurrence pour Mersjohann, cela est vrai, n'est qu'une partie infime de toutes les trahisons, duperies, reniements et compromis qui sont au cœur du commerce entre les hommes. Mersjohann a soutenu une thèse de doctorat sur "Vérité et mensonge chez Kleist». C'est dire qu'il sert strictement à rien d'être dans son bon droit et de se raidir dans ses exigences, il ne parvient pas à cultiver une saine dose d'indifférence à l'égard de la perfidie avec toutes ses variantes. Pour lui, la trahison détruit notre être. La trahison « te pulvérise », dit-il (zerreibt dich). Même si on tuait ceux qui nous démolissent, cela ne changerait pas grand-chose, car ce qu'ils ont démoli est bel et bien démoli... Mersjohann est capable d'entrer dans le monde des apparences et d'y jouer le jeu, mais il n'a pas de défense contre le monde de l'hypocrisie, en somme contre le monde tout court. Aussi en sort-il à reculons. Sans être vraiment un moraliste, il ne respire que dans ces zones marginales où la fragilité de l'être, sa sensibilité, son discernement aussi, ne constituent pas immédiatement la cible des hommes infaillibles et vertueux qui, de préférence au nom d'un principe noble, écartant de leur chemin ascendant tous les Mersjohann dès que ceux-ci risquent d'entraver les stratégies savantes des champions de la manipulation.

Dans le système du «publish or perish», des qualités de poète ne sont pas indispensables. Et si la compétence reste un critère valable, les scrupules ne doivent pas être excessifs. Où en est-il, Mersjohann, avec ses travaux, personnels et scientifiques ? Un jour, Halm saisit l'occasion de lui demander s'il écrivait encore. Mersjohann, 
visiblement touché, garde quand même son calme et remercie Halm de sa question, " une vraie question pour Amfortas » (eine Amfortas-Frage). Puis il ajoute qu'ils allaient bientôt dîner « chez le poète de service » (beim Dichter vom Dienst), et qu'on y évoquerait assez cette vaine occupation. Avec cette remarque un peu sèche, le sujet est épuisé. En a-t-il jamais parlé à sa femme ? Au cours de l'histoire nous apprenons qu'Elissa n'est pas une passionnée de lecture et qu'elle ne sait pas l'allemand. Quant à Mersjohann, il s'exprime la plupart du temps en anglais, tout en passant rapidement d'une langue à l'autre lorsqu'il s'agit de faire comprendre une expression idiomatique à ses amis européens ou à leur résumer l'essentiel d'une conversation. "Ce pays est un pays de mots ", dit-il de la Californie où les gens, selon lui, étaient "complètement fous des mots » (ein Wörterland; die Leute seien ganz wild auf Wörter). « Ici, les mots arrangent tout, en toutes circonstances. Il n'y avait donc plus de circonstances » (Die Wörter erledigten die Sachen, hier. Es gebe dann keine Sache mehr). Dans cet univers de mots, Mersjohann se tait souvent, allumant une cigarette après l'autre, ce qui lui permet de se retirer afin que la fumée ne soit pas désagréable à ses hôtes. Evidemment, il y a des silences éloquents, mais dans un pays de mots, c'est le savoir-parler qui compte et c'est parfois la volubilité qui suffit. Mersjohann, le taciturne, Mersjohann, le poète, «le jeune génie » d'autrefois, n'avait-il pas confié à l'écriture son mal d'être? Le jour où Mesjohann aura disparu, Lena Halm recevra un paquet qui contient un manuscrit: California Fragment. 154 sonnets. Des sonnets d'amour, composés pour Elissa. Nous n'en saurons pas plus.

On connaît les remarques célèbres de Baudelaire sur les bienfaits du travail, sous la rubrique « Hygiène » dans ses Journaux intimes:

Plus on travaille, mieux on travaille, et plus on veut travailler. Plus on produit, plus on devient fécond. Pour guérir de tout, de la misère, de la maladie et de la mélancolie, il ne manque absolument que le goût du travail.

Mersjohann est auteur de nombreux articles. Il fait partout des conférences brillantes, soigneusement préparées. Bientôt il prendra l'avion pour Houston où il parlera de Schubert, plus précisément de la Compréhension des textes de Schubert (Schuberts Textverständnis). À l'Université, il s'installe d'arrache-pied. Mais les semaines passent et Mersjohann n'a pas encore écrit une seule ligne de cet essai dont il dit par ailleurs qu'il paralysait tout le reste de son activité. Où va-t-on avec cette contrainte de rédiger bêtement des séries d'essais ... (Dieser Zwang, sinnlos Aufstatz um Aufsatz zu schreiben...). Voyons un peu ce professeur de philosophie, Felix Theodor Auster, dont la productivité est étonnante et sûrement intarissable (à moins que Auster, lui aussi, ne meure comme nous tous, encore que cela paraisse inconcevable) : vous écrivez un livre sur Nietzsche? Auster aussi. Vous écrivez un livre sur Kafka? Auster est justement en train de corriger les épreuves de son étude Nietzsche d'abord, et puis Kafka. À Halm, il donnera son petit opuscule sur la Morosité conservatrice chez Heine (Über konservativen Unmut bei Heine), et à Mersjohann il avait envoyé son essai Le Lied philosophique, ou Que savait Schubert avant Schopenhauer? (Das philosophische Lied oder Was wußte Schubert vor Schopenhauer?). Publish or perish. Et ne pas oublier de citer les travaux de vos collègues, de ceux seulement, bien entendu, qui citent les vôtres.

Mersjohann n'avance plus dans son travail. En revanche, le temps, lui, ne s'arrête pas. Vient le moment où il doit se rendre à Houston. La secrétaire à Oakland manifeste de l'inquiétude : Mersjohann part sans manuscrit, tout au moins il ne lui avait pas remis de texte à taper, ce qu'il avait fait jusqu'alors, ses communications étant toujours fignolées, elle en savait quelque chose, même s'il pouvait ensuite donner l'impression 
d'improviser, de trouver sur-le-champ une formule heureuse... Cette fois-ci, il se présente sans manuscrit à un congrès auquel participaient des personnalités venues de toute l'Amérique. «Que fera-t-il donc? » demande-t-elle. qu'une feuille, rien qu'une phrase :

Et il serait peu convenable, messieurs, à l'âge qui est le mien, que je me présente devant vous comme un petit jeune homme avec un discours tout fait.

Felix Theodor Auster reconnaît tout de suite la citation, tirée de L'Apologie de Socrate de Platon, dans la traduction de Schleiermacher (Auch würde es sich ja schlecht ziemen, ihr Männer, in solchem Alter gleich einem Knaben, der Reden vorbereitet, vor euch hinzutreten).

De «publish or perish», Mersjohann avait choisi «perish». Il ne faisait pas partie des vedettes au visage dur, splendide et audacieux, il n'était pas de la paroisse des êtres éminents aux articulations souples. "On se perd", avait-il dit (man geht eben verloren). Et puis, en s'adressant à Halm : «take care». Martin Walser sait à la perfection suggérer l'inéluctable.

\section{NOTES}

1. - Martin Walser, Brandung, Frankfurt/Main, Suhrkamp Verlag, 1985.

Nous avons utilisé, avec quelques modifications, la traduction d'Hélène Belletto : Ressac, Paris, Laffont, 1987.

\section{RÉSUMÉS}

Martin Walser, l'un des plus subtils critiques de son temps, mais aussi un psychologue averti et cultivé, analyse dans Ressac les milieux universitaires de la côte californienne. Il observe les travers d'une société d'intellectuels, il pénètre les secrets de l'âme humaine, perçoit les espoirs, les attentes, les déceptions. Avec Rainer Mersjohann, un professeur d'origine allemande qui vit aux États-Unis, il témoigne de la naissance et du développement d'un mal d'être qui conduit à une fin tragique : un homme court à sa perte, une vie est ratée.

Martin Walser, einer der scharfsinnigsten Kritiker seiner Zeit, aber auch ein kluger und geschulter Psychologe, analysiert in Brandung das Universitätsmilieu an der californischen Küste. Er beobachtet die Mißstände in den Kreisen der Intellektuellen, er dringt in die Geheimnisse der menschlichen Psyche ein, erkennt Hoffnungen, Erwartungen, Enttäuschungen. Mit Rainer Mersjohann, einem Professor deutscher Herkunft, der in den Vereinigten Staaten lebt, zeigt er 
Entstehung und Entwicklung eines existentiellen Unbehagens, das zu einem tragischen Ende führt: ein Mensch richtet sich zugrunde, ein Leben ist verfehlt.

\section{AUTEUR}

\section{ERIKA TUNNER}

Université Charles de Gaulle, Lille III 